\title{
Novel Drug Candidates for the Treatment of Metastatic Colorectal Cancer through Global Inverse Gene-Expression Profiling
}

\author{
Vera van Noort ${ }^{1,2}$, Sebastian Schölch ${ }^{3}$, Murat Iskar ${ }^{1}$, Georg Zeller ${ }^{1}$, Kristina Ostertag ${ }^{4}$, \\ Christine Schweitzer ${ }^{3}$, Kristin Werner ${ }^{3}$, Jürgen Weitz ${ }^{3}$, Moritz Koch ${ }^{3}$, and Peer Bork ${ }^{1,5}$
}

\begin{abstract}
Drug-induced gene-expression profiles that invert disease profiles have recently been illustrated to be a starting point for drug repositioning. In this study, we validate this approach and focus on prediction of novel drugs for colorectal cancer, for which there is a pressing need to find novel antimetastatic compounds. We computationally predicted three novel and still unknown compounds against colorectal cancer: citalopram (an antidepressant), troglitazone (an antidiabetic), and enilconazole (a fungicide). We verified the compounds by in vitro assays of clonogenic survival, proliferation, and migration and in a subcutaneous mouse model. We found evidence that the mode of action of these compounds may be through inhibition of TGF $\beta$ signaling. Furthermore, one compound, citalopram, reduced tumor size as well as the number of circulating tumor cells and metastases in an orthotopic mouse model of colorectal cancer. This study proposes citalopram as a potential therapeutic option for patients with colorectal cancer, illustrating the potential of systems pharmacology. Cancer Res; 74(20); 5690-9. (C)2014 AACR.
\end{abstract}

\section{Introduction}

Rational drug discovery has gained momentum by the recently increased availability of large-scale datasets on biologic activities of small molecules. Integration and utilization of these heterogeneous resources require computational tools, as well as expert knowledge to guide the drug discovery or repositioning process. To enable the latter, a pioneering systems pharmacology study built the Connectivity Map (CMap), a collection of genome-wide gene-expression readouts of cell lines treated with more than 1,000 drug-like chemicals (1). This approach is fundamentally based on the observation

${ }^{1}$ Structural and Computational Biology Unit, European Molecular Biology Laboratory, Meyerhofstrasse, Heidelberg, Germany. ${ }^{2}$ Centre of Microbial and Plant Genetics, KU Leuven, Kasteelpark Arenberg, Leuven, Belgium. ${ }^{3}$ Department of Gastrointestinal, Thoracic and Vascular Surgery, Medizinische Fakultät Carl Gustav Carus, Technische Universität Dresden, Dresden, Germany. ${ }^{4}$ Department of General, Gastrointestinal and Transplant Surgery, University Hospital Heidelberg, University of Heidelberg, Heidelberg, Germany. ${ }^{5}$ Max-Delbrück-Centre (MDC) for Molecular Medicine, Berlin, Germany.

Note: Supplementary data for this article are available at Cancer Research Online (http://cancerres.aacrjournals.org/)

V. van Noort, S. Schölch, and M. Iskar contributed equally to this article.

Corresponding Authors: P. Bork, European Molecular Biology Laboratory, meyerhofstrasse 1, Heidelberg, Germany 69117. Phone: 49-6221387-8361; Fax: 49-6221-387-8517; E-mail: bork@embl.de; and M. Koch, Department of Gastrointestinal, Thoracic and Vascular Surgery, Medizinische Fakultät Carl Gustav Carus, Technische Universität Dresden, Dresden, Germany. Phone: 49-351-458-2863 Fax: 49- 351-458-4317; E-mail: Moritz.Koch@uniklinikum

doi: 10.1158/0008-5472.CAN-13-3540

(C)2014 American Association for Cancer Research. that, in many cases, drugs with similar mechanism of action in vivo also elicit similar expression responses in this in vitro model system, thus providing a starting point for drug repositioning based on expression profile similarity $(1,2)$. Moreover, it was proposed that a drug-induced gene-expression profile that (partially) inverts a disease-associated expression profile could hint at a potential treatment for the respective disease and indeed, by applying this concept, novel compounds have been recently proposed for inflammatory bowel disease, non-small cell lung cancer, and muscle atrophy (3-6). Thus, the repositioning of drugs based on specifically tailored gene signatures has been demonstrated for these diseases. However, although an enrichment of drug-disease relations has been found among anticorrelated profiles when all diseases are considered together, it remains to be investigated how well this approach works for each individual disease where an expression profile is available.

A systematic analysis requires addressing various biases that are inherent to the CMap data, such as batch effects, which can be partially removed with a normalization procedure (7). Furthermore, to predict new treatment candidates for a disease, the disease itself needs to be clearly defined and characterized at the molecular level. Finally, a robust gene-expression profile, associated with the disease that is also relevant for the disease etiology, is necessary to make accurate predictions.

Here, we present a global, unbiased approach for using drug-induced gene-expression profiles to retrieve novel candidate drugs with many cancer profiles being inversely matched. Our analysis shows a particularly strong signal for colorectal cancer, which is the third most common cancer in Western countries (8). Patients with colorectal cancer with 
nonmetastatic disease have excellent long-term survival as in most cases localized primary tumors can be surgically removed. However, survival rates drop significantly with the occurrence of distant metastases. Although hepatic metastases can be surgically removed in a curative intention, thereby providing long-term cure in a significant number of patients, metastatic disease often recurs, and patients succumb to recurrent metastatic disease or primarily unresectable multifocal metastases $(9,10)$. The fate of patients with colorectal cancer is therefore closely linked to the occurrence of distant metastases. Although the clinical significance of metastasis in colorectal cancer is evident, the process of metastasis is still poorly understood (11). It is widely accepted that circulating tumor cells play a pivotal role in distant tumor dissemination and their detection is closely linked to the prognosis of patients with colorectal cancer (12-16). To actively leave the tumor, enter the blood stream, attach to the vessel endothelium in the target organ, invade, proliferate, and form new lesions, abilities far beyond those of a bulk tumor cells (e.g., migration and invasion capacities) are essential for metastatic tumor cells. In that respect, to reduce the risk of (further) tumor cell dissemination in patients with colorectal cancer, especially in the neoadjuvant situation (e.g., in locally advanced rectal cancer requiring neoadjuvant treatment or the "liver first" approach in synchronously metastasized colorectal cancer; ref. 17), there is a huge clinical need for (novel) antimetastatic drugs.

We illustrate the power of our approach by experimentally validating three predicted novel drugs that could be repositioned for colorectal cancer. We specifically test the three predicted drugs for their potential to inhibit metastasis. We confirm in vitro that these drugs inhibit cell migration. Second, we validate that they inhibit tumor growth in vivo in a subcutaneous mouse model. Finally, using an orthotopic mouse model of colorectal cancer, we confirm the antimetastatic properties of citalopram in vivo. Taken together, from a broad and comprehensive bioinformatics approach, via various computational and experimental filtering and validation procedures, we arrived at a very specific, clinical relevant indication in the context of colorectal cancer and propose that the antidepressant drug citalopram can serve at least as a lead for treatment of metastatic colorectal cancer.

\section{Materials and Methods}

\section{CMap data}

The CMap (build 02) is a large-scale microarray resource cataloguing transcriptomic responses of four human cell lines (promyelocytic leukemia HL60, breast adenocarcinoma MCF7, prostate cancer PC3, and skin melanoma SKMEL5 cell lines) to various small-molecule treatments (referred to as drugs for simplicity). It contains 6,100 treatment instances for 1,309 unique drugs, of which approximately 650 are FDA approved $(1,18)$. To eliminate various biases in CMap (e.g., batch effect, microarray platforms), we filtered and normalized the raw CMap dataset (downloaded from http://www.broadinstitute.org/cmap/) as described in detail previously (7). For this study, we retrieved 4,849 druginduced gene-expression profiles from three main cell lines
(HL60, MCF7, and PC3) representing 1,144 distinct drugs (for a detailed list, refer to Supplementary Table S3).

\section{Disease-associated microarrays}

Microarray experiments on specific diseases were identified in the public microarray repository of NCBI Gene Expression Omnibus (GEO; http://www.ncbi.nlm.nih.gov/geo/; ref. 19) using disease medical subject heading (MeSH) terms that were attributed to each study (20-22). In this study, we restricted our analysis to disease MeSH terms that are associated with at least one CMap drug. Moreover, we only analyzed raw datasets conducted on HG-U133A and HG-U133-Plus2 microarray platforms to maximize comparability across resources. Next, all individual samples were manually annotated as "disease" or "healthy controls." To obtain reliable disease signatures, microarray samples (disease vs. healthy) were retained only if derived from disease-relevant primary tissues. The RMA procedure was employed to normalize each dataset separately (23). In cases, where multiple studies were found for the same disease, we selected one representative per disease, such that distinction between disease and healthy samples is maximized on the basis of interarray Pearson correlation (highest AUC in receiver operating characteristic analysis), and discarded the others. Finally, in each disease-related geneexpression profile, only probe sets present in HT_HG-U133A (HT Human Genome U133 Array Plate Set, 22277 probe sets) were kept and ranked according to their fold change. In total, we obtained here a total of 40 disease-associated gene-expression profiles from 1,748 individual microarray samples (for details refer to Supplementary Table S1).

\section{Similarity score of drugs and diseases}

To calculate profile similarity of a drug with a disease, we adapted a profile comparison method initially developed for drug-drug comparisons (7). First, a disease-related signature was defined from the top and bottom 250 ranked probe sets of drug-associated gene-expression profiles. We then computed disease-drug similarity by querying the up- and downregulated signatures of the disease within drug-induced geneexpression profiles (HT_HG-U133A, 22277 probe sets without Present/Absent call filtering) using gene set enrichment analysis (GSEA, weighted; ref. 24). At last, two enrichments scores for up/down disease signatures were averaged to derive a final score for disease-drug similarity. We assessed whether known drug-disease associations (indications, excluding "antidotes" from http://www.drugs.com/medical_conditions.html updated October 7, 2013) are significantly anticorrelated using a nonparametric Wilcoxon signed rank test. To this end, similarity scores from each cell line were ranked in the range of $(0-1)$ and pooled together. For each disease, we compared the similarity scores of known drug associations to all other drugs present in CMap (Supplementary Fig. S1 and Supplementary Table S2). Significantly, anticorrelated diseases were defined as $P<0.05$ after FDR correction for multiple testing.

\section{Metastatic colon cancer gene signature}

To identify novel therapeutics against metastatic colorectal cancer, we utilized a consensus gene signature defined by 
Jorissen and colleagues (25). From two independent sets of colorectal cancers, a metastatic signature was derived that showed consistent expression changes between early-stage (A) and metastatic stage (D) tumors. In total, there were 86 stage A tumors, 91 stage D primary tumors, and 30 stage $\mathrm{D}$ metastases. The signature was derived from consistent changes between three comparisons: (i) early-stage (A; $n=$ $44)$ and metastatic stage $(D ; n=61)$ primary tumors from the first independent set; (ii) early-stage (A; $n=42)$ and metastatic stage (D; $n=32)$ primary tumors from the second set; and (iii) early-stage (A; $n=42)$ primary tumors and metastatic stage $(\mathrm{D} ; n=30)$ metastases from the second set. The signature consists of 163 probe sets representing in total 127 unique genes (73 up- and 54 downregulated; Supplementary Table S3). Probe sets not present in the CMap dataset (HT_HG-U133A platform) were removed from the signature. After filtering, the metastasis signature was compared against the CMap reference collection that contains 4,849 gene-expression profiles from three cell lines treated with 1,144 distinct drugs. We used the scoring approach described above to quantify profile similarity between drugs and the metastasis signature. For each drug, a final score was obtained by averaging the enrichment scores across all replicates from three cell lines (Supplementary Table S4). We further examined the top 20 drugs with lowest scores (anticorrelated) and manually searched in the literature whether these drugs were previously associated with colon cancer or metastasis.

\section{Cell lines and reagents}

The colorectal cancer cell lines, HCT 116 and HT-29, were obtained from ATCC and maintained in DMEM (PAA) + 10\% FCS (PAA), $100 \mathrm{U} / \mathrm{mL}$ penicillin (PAA), and $100 \mu \mathrm{g} / \mathrm{mL}$ streptomycin (PAA) in a humidified atmosphere of $5 \% \mathrm{CO}_{2}$ at $37^{\circ} \mathrm{C}$. The cell lines were tested for authenticity by DSMZ on a regular basis. Enilconazole (Imazalil) and troglitazone were obtained from Sigma-Aldrich and Neochema, citalopram was obtained from Lundbeck.

\section{MTT proliferation assay}

The WST-1 reagent (Roche) is reduced by metabolically active cells to formazan, an insoluble purple dye that can be measured in a spectrophotometer, and was used according to the manufacturer's instructions. Cells were seeded in quadruplicates in increasing cell numbers in 96-well plates $(125,250$, $500,1,000$ per well). After 12-hour adhesion time, the cells were treated with the compounds as indicated. One hundred and twenty hours later, $10 \mu \mathrm{L}$ of WST-1 reagent were added to each well and the cells were again incubated for 4 hours at $37^{\circ} \mathrm{C}$ and $5 \% \mathrm{CO}_{2}$. After incubation and 1 minute shaking, the absorbance was measured on a Genios Microplate Reader (Tecan).

\section{Clonogenic survival assay}

Clonogenic survival assay was done as previously described $(26,27)$. In brief, tumor cells were plated in triplicates to yield 50 to 100 colonies per culture flask, treated with the compounds in various concentrations, and incubated for 14 to 21 days. Colonies of more than 50 cells, as assessed by microscopic inspection, were scored as survivors and counted.

\section{Migration assay}

The migration of tumor cells under treatment was measured via Transwell assays as described previously $(26,27)$. Briefly, cells were incubated with the compounds in various concentrations for 2 hours. Then $200 \mu \mathrm{L}$ of cell suspension $(3 \times 105$ cells $/ \mathrm{mL})$ were added in triplicate Transwells (Matrigel-coated Transwell inserts; $8 \mu \mathrm{mol} / \mathrm{L}$ pore size; Becton Dickinson). After 18 hours of incubation, migratory tumor cells had invaded the underside of the membrane, were fixed, stained in thiazine and eosin solution, and sealed on slides. Quantification was done by microscopic counting.

\section{TGF $\beta$ signaling assay}

To demonstrate the effects of the three compounds on the TGF $\beta$ signaling pathway, we used a cell line stably expressing a secreted alkaline phosphatase reporter inducible by SMAD3/4inducible elements (SBE), called HEK-Blue TGF $\beta$ sensor cell line (Invivogen). This way, downstream activation of the TGF $\beta$ signaling pathway can be measured spectrophotometrically in the cell culture supernatants. The effects of the three compounds on TGF $\beta$ signaling pathway were analyzed using the HEK-Blue TGF $\beta$ Kit (Invivogen) according to the manufacturer's instructions.

\section{Animal experiments}

All animal experiments strictly adhered to local and federal regulations as well as FELASA guidelines, and were approved by the local authorities before initiation.

For the subcutaneous experiments, $10^{7}$ tumor cells in $100 \mu \mathrm{L}$ of PBS were injected bilaterally into the flanks of NOD.Cg$P r k d c^{\text {scid }} I l 2 r g^{\text {tml Wjl }} / \mathrm{SzJ}$ (NSG) mice. When tumors reached a size of 10 to $12 \mathrm{~mm}$ in largest diameter, the mice were euthanized, the tumors excised, and cut into tumor fragments of $1 \times 1 \mathrm{~mm}$ size. The tumor fragments were implanted subcutaneously into recipient NSG mice and monitored thrice weekly for tumor growth. Once tumors were established, growing, and had a size of 7 to $8 \mathrm{~mm}$ in largest diameter, the treatment was initiated.

For orthotopic tumor cell injection, the mice were anesthetized by isoflurane inhalation. A midline laparotomy was performed and the cecum was exteriorized and $10^{5} \mathrm{HCT}$ 116 tumor cells in $20 \mu \mathrm{L}$ Matrigel (BD) were injected subserosally by help of a microinjection pump (WPI) under microscopic visual control. After injection, the abdomen was closed with PDS II 6-0 (J\&J Ethicon) and wound clips.

The treatment regimens were as follows:

Citalopram: $60 \mathrm{mg} / \mathrm{kg}$ body weight (BW) i.p. daily, dissolved in $100 \mu \mathrm{L} \mathrm{NaCl} 0.9 \%$ in the subcutaneous experiment. Because of low tolerability (significant weight loss due to loss of appetite), the dose was reduced to $20 \mathrm{mg} / \mathrm{kg}$ i.p. daily for the orthotopic experiment.

Enilconazole: $20 \mathrm{mg} / \mathrm{kg}$ BW i.p. daily, dissolved in $20 \mu \mathrm{L}$ DMSO

Troglitazone: $30 \mathrm{mg} / \mathrm{kg}$ BW i.p. every third day, dissolved in $100 \mu \mathrm{L} 9 \%$ Solutol HS 15 


\section{Quantification of circulating tumor cells}

Thirty-five days after orthotopic tumor cell injection, the mice in the control group were moribund and the experiment was terminated. The mice were anesthesized by isoflurane inhalation, blood samples were taken via intracardiac puncture, and the mice were euthanized. The blood was layered over LSM 1077 lymphocyte gradient medium (PAA) and the peripheral blood mononuclear cell (PBMC) fraction was enriched according to the manufacturer's instructions. The resulting PBMCs were stained with Alexa-Fluor488-anti-human EpCAM antibody (Biolegend), EpCAM-positive cells were identified and counted under a fluorescence microscope (Leica). The number of circulating tumor cells (CTC) per mL of blood was calculated according to the total blood volume obtained by cardiac puncture.

\section{Histologic work-up}

Upon euthanasia, the livers and lungs of the animals were removed and immediately fixed in $4 \%$ paraformaldehyde. After paraffin embedding, the FFPE tissue was cut in $10 \mu \mathrm{m}$ (liver) or $2 \mu \mathrm{m}$ (lung) sections using a Leica microtome. Lungs were cut in a representative layer in the coronal plane; livers were cut in three representative layers (cranial third, middle third, and caudal third) in the transversal plane to be able to detect the majority of metastatic lesions. The tissue sections were stained with a standard hematoxylin and eosin protocol and metastases were counted.

\section{Statistical analysis}

Datasets were screened for outliers with the ROUT method $(\mathrm{Q}=10 \%$; ref. 28$)$, a total of four statistically significant outliers were identified (all in the orthotopic citalopram experiment: 1 among the tumor weight values, 2 among the tumor volume values, 1 among the CTC number values). The findings were checked for biologic validity and the values were excluded when the cause of the deviation was considered artificial (e.g., faulty measurements). Three out of 4 outliers were found to be biologically invalid values and excluded from further analysis; the outlier among the CTC values was considered biologically possible and was therefore not excluded. Student $t$ test was used to compare means. In case of time series, only the last time-point was tested. All analyses were two tailed, and $P<0.05$ was considered statistically significant $\left({ }^{*}, P<0.05\right.$; ${ }^{* *}, P<0.01$; $\left.{ }^{* * *}, P<0.001\right)$.

\section{Propidium iodide/Annexin $\mathrm{V}$ apoptosis assay}

A total of $5 \times 10^{4}\left(\right.$ HT-29) or $3 \times 10^{4}$ (HCT116) colorectal cancer cells per well were seeded in 24-well plates, allowed to adhere for 12 hours, and then treated with the compounds, negative or positive controls as indicated. Seventy-two hours after incubation, the cells were harvested with trypsin and washed twice with FACS-binding buffer ( $10 \mathrm{mmol} / \mathrm{L}$ Hepes, $\mathrm{pH}$ $7.4,2.5 \mathrm{mmol} / \mathrm{L} \mathrm{CaCl} 2,140 \mathrm{mmol} / \mathrm{L} \mathrm{NaCl})$. Then the cells were stained with propidium iodide (PI) and Annexin V-FITC (Becton Dickinson) and 10,000 cells were analyzed and quantified by flow cytometry on a FACS Calibur (Becton Dickinson). Annexin-positive cells were considered apoptotic and the ratio of apoptotic cells/total cells was calculated.

\section{Results}

\section{Reverse profile searches for retrieval of drug-disease associations}

To evaluate the general validity of the inverse signature approach, we assessed how well the known disease-drug indications were recapitulated by the expression-based inverse correlation of disease-drug relations for individual diseases. To this end, we first normalized the CMap dataset to obtain 4,849 drug-induced gene-expression profiles from three main cell lines (HL60, MCF7, and PC3) treated with 1,144 distinct drugs (7). In addition, we extracted diseaseassociated microarray experiments from publicly available NCBI GEO microarray repository using MeSH descriptors (19). For this analysis, we next generated disease-associated gene-expression profiles for 40 distinct diseases (details in Materials and Methods).

Indeed, in the normalized CMap data, significant anticorrelations were found between drug and disease gene-expression profiles for known drug-disease associations for seven out of 40 diseases (Supplementary Fig. S1). Anticorrelations were most successful in the retrieval of disease-drug relations involving anticancer agents that were additionally linked to multiple cancers (six out of seven significant diseases), including colorectal cancer (Supplementary Fig. S1). On the basis of this result, we focused on colorectal cancer to predict novel drug compounds aiming specifically at an inhibition of metastasis as the latter is of considerable clinical relevance.

\section{Prediction of novel drug compounds for metastatic colorectal cancer}

As colorectal cancer scored very prominently among the general disease-drug relations and because there are insufficient treatment options, particularly for inhibiting metastasis, we chose to illustrate the power of the inverse-signature method by predicting and validating drugs used in other indications as candidates for colorectal cancer treatment. Several gene-expression signatures for colorectal cancer are available, but the most pressing need is for drugs that inhibit metastasis, which could also be used as an adjuvant therapy. As opposed to previous studies that have used healthy versus diseased states (29), we rather chose to compare nonmetastatic tumors versus metastatic primary tumors and metastases, to define a gene signature of metastatic potential rather than a general cancer signature. Several groups have studied the gene-expression differences between primary and metastatic colon cancers to explain the molecular basis of the metastatic process and predict clinical outcomes $(25,30-$ 32 ). In this study, we decided to employ the reproducible gene-expression signature from (25) that emerged as a consensus from two independent comparisons of Dukes' stage D versus stage A colorectal cancers (Supplementary Table S3; for details see Materials and Methods). This signature is based on primary and metastatic tumors from patients in different stages of colorectal cancer and includes genes that have been implicated in the development of metastasis before. We predict novel antimetastatic drugs by finding anticorrelated druginduced gene-expression profiles, that is, we are looking for genes that are upregulated in the metastasis profile, but 


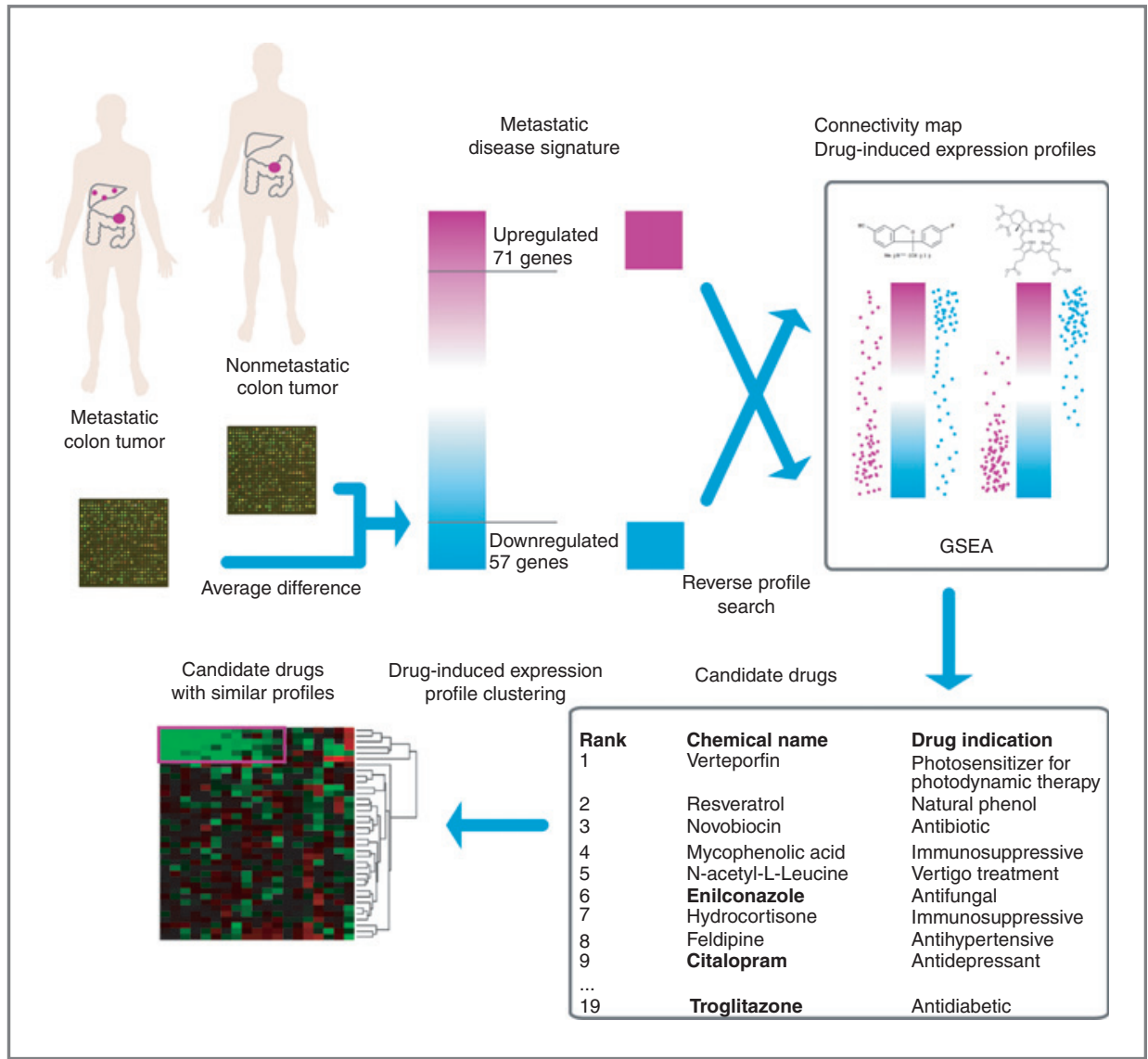

Figure 1. Using anticorrelated profiles to find candidate therapeutic chemicals. First, a metastatic disease signature was created by comparing metastatic colorectal cancers with nonmetastatic cancers. The disease signature was then used to search for reverse signatures in the CMap data by Gene Set Enrichment Analysis. The resulting candidate drugs do not necessarily have similar gene-expression profiles; for example, different subsets of upregulated genes from the disease signature can be downregulated in the druginduced expression profile. Therefore, an extra refinement step was added by clustering the expression profiles of candidate drugs and selecting from the candidates those that have similar gene profiles for further validation.

downregulated by adding a drug to one of the three CMap cell lines (Fig. 1).

The validity of the procedure is illustrated by the fact that among the top chemicals with inverse drug-induced geneexpression profiles, compared with the metastatic colon cancer gene signature (Fig. 1 and Supplementary Table S4), were verteporfin (33), resveratrol (34), and novobiocin (35), all of which have already been shown to inhibit migration and/or the metastatic development of cancer cells (Table 1; refs. 33-35). Upon drug treatment by any of these three drugs (Fig. 2), fibronectin 1 , collagen type $\mathrm{V}$, notch homolog 3 , platelet-derived growth factor $C$, versican, integrin- $\beta 5$, and secreted phosphoprotein 1 are downregulated. Four of the proteins encoded by these genes are involved in interactions between the extracellular matrix (ECM) and ECM-receptors (Supplementary Fig. S2). The proteins fibronectin 1 and its

Table 1. Per cell line best inverse signature GSEA scores (-1 to 1$)$ for candidate and proposed drugs

\begin{tabular}{lllllll} 
Small molecule & PUBCHEM ID & CMap cell line & CMap batch & CMap experiment ID & GSEA score \\
\hline Verteporfin & 5362420 & MCF7 & 678 & $5500024028849050407172 . F 09$ & -0.58 \\
Enilconazole & 37175 & HL60 & 661 & $5500024030700072107987 . B 09$ & -0.57 \\
N-acetyl-L-leucine & 1995 & HL60 & 660 & $5500024030700072107987 . E 06$ & -0.53 \\
Troglitazone & 5591 & PC3 & 727 & $5500024031723100807773 . C 04$ & -0.47 \\
Resveratrol & 5056 & HL60 & 640 & $640641112706 . G 01$ & -0.46 \\
Felodipine & 3333 & HL60 & 634 & $5500024024214122006604 . F 03$ & -0.45 \\
Mycophenolic acid & 4272 & MCF7 & 690 & $5500024030403071907257 . B 06$ & -0.40 \\
Citalopram & 2771 & MCF7 & 720 & $5500024031723100807770 . B 03$ & -0.39 \\
Novobiocin & 4545 & HL60 & 658 & $5500024030760072207028 . G 04$ & -0.38 \\
Hydrocortisone & 3640 & PC3 & 714 & $5500024031723100807776 . H 03$ & -0.37
\end{tabular}

NOTE: Proposed drugs are in bold. 
receptor integrin- $\beta 5$ together are vital for cell migration (36). This confirms the metastatic colorectal cancer gene signature to be appropriate for our purpose. The candidate drugs found by inverse profile search do not fully overlap in the set of genes whose expression is inverted relative to the disease profile. Thus, to find the most relevant drug candidates, we refined our list of top 20 candidate compounds by hierarchical clustering of the drug-induced gene-expression data from individual experiments in different cell lines and batches (Fig. 1) and continue with those that cluster together (Fig. 2). The clustering shows that some marketed drugs such as enilconazole (antifungal), citalopram (antidepressant), and troglitazone (antidiabetic) induce regulatory responses similar to verteporfin and resveratrol (Fig. 2).

The best scoring candidate drugs, namely enilconazole, citalopram, and troglitazone, were selected for further experimental validations. Among these, only troglitazone (an antidiabetic and anti-inflammatory drug) has been previously associated with decreased migratory behavior of various cell types (37-39) but not with colorectal cancer. For example, troglitazone has been screened in breast cancer metastasis. It has been reported that troglitazone reduces the migration, adhesion, and spreading of human breast cancer cells on fibronectin (FN)-coated plates independent of its PPAR $\gamma$ action $(37,39)$ and inhibits pulmonary metastasis of osteosarcoma (38). Second, citalopram, an antidepressant drug of the selective serotonin reuptake inhibitor class is approved to be used in humans but not an obvious candidate for screening against metastatic colorectal cancer. The third candidate enilconazole is a fungicide that has only been approved for topical use in veterinary medicine. Hence, the three candidates to be validated experimentally for their efficacy against metastatic colorectal cancer are not only chemically diverse, but also come with different levels of expectation.

\section{Mode of action}

To better understand the underlying basis of our predictions, we set out to find the main mode of action of the three selected compounds. There are various signaling pathways that could play a role. One of them, TGF $\beta$ exerts its effects on cell proliferation, differentiation, and migration in part through its modulation of extracellular matrix components, such as fibronectin and plasminogen activator inhibitor-1 (40), which we find downregulated by the candidate drugs. It has recently been shown that resveratrol inhibits the TGF $\beta 1$ induced increase in cell adhesion, migration, and invasion

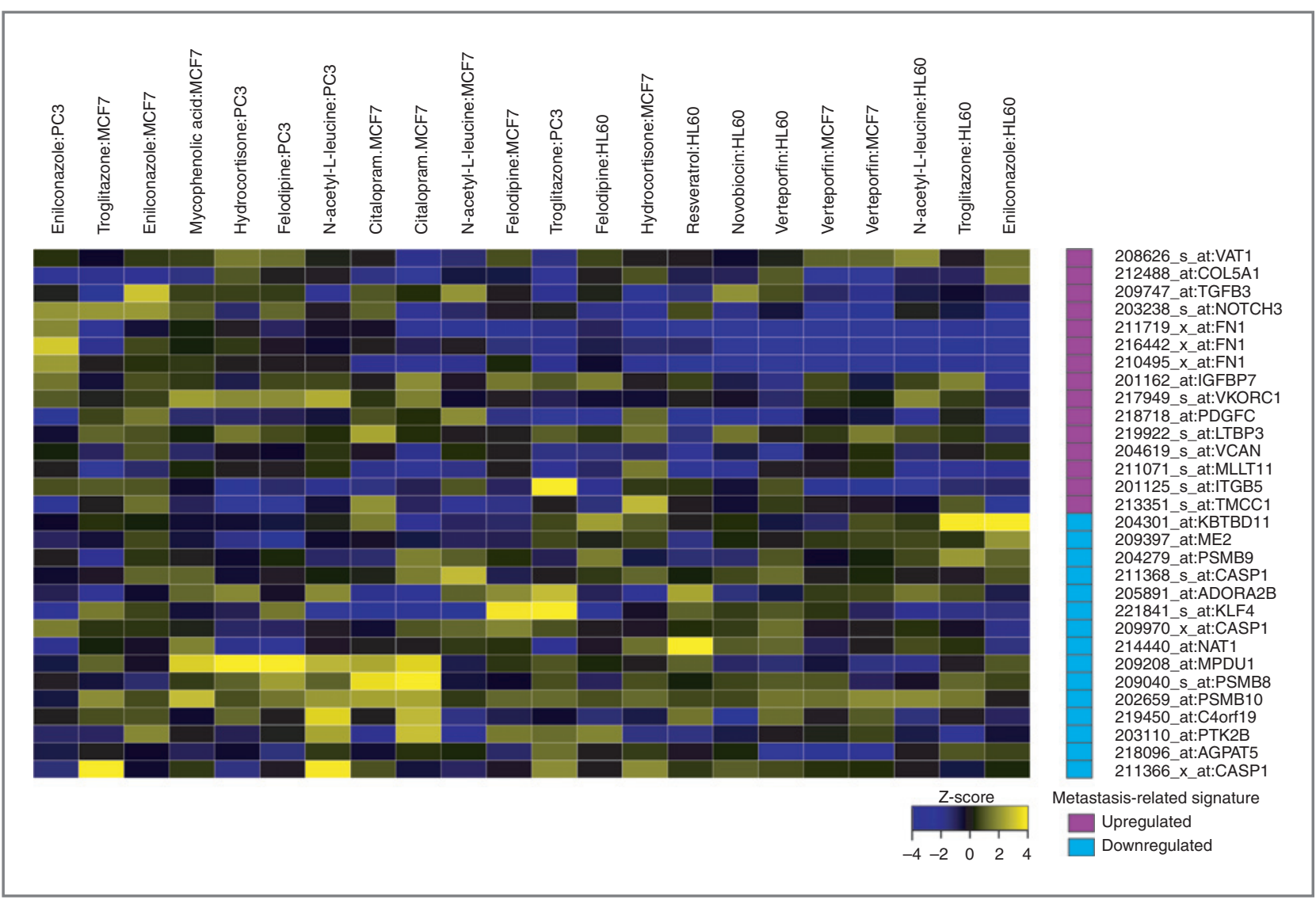

Figure 2. Evaluation of gene-expression profile clustering of candidate chemicals. Clustering of CMap data of individual experiments of 10 refined drug candidates (most anticorrelated profiles per cell line) and top 30 anticorrelated genes. Purple, upregulated in metastatic colorectal cancer signature; blue, downregulated in metastatic colorectal cancer signature. Dark blue, normalized fold changes, downregulated in drug-induced profile; yellow, upregulated in the drug-induced profile. 


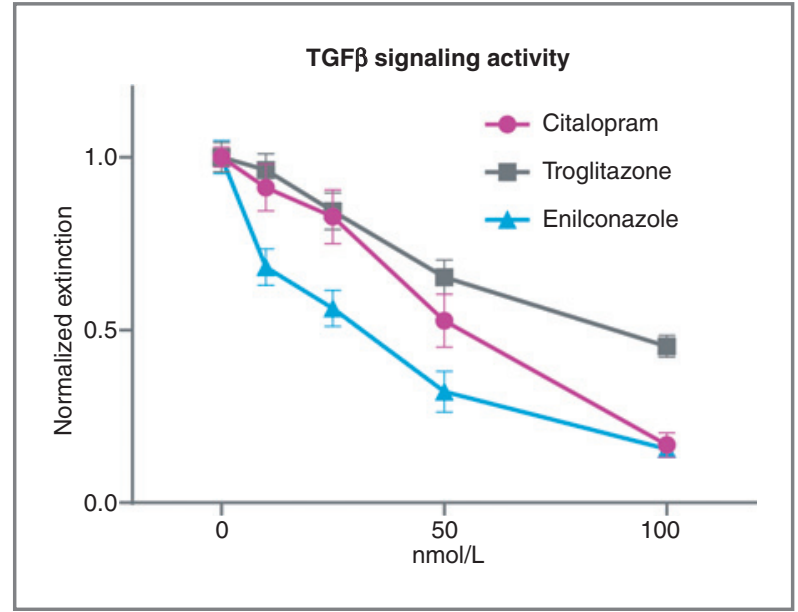

Figure 3. Mode of action of candidate chemicals. Normalized TGF $\beta$ signaling activity with different concentrations of citalopram, troglitazone, and enilconazole. Mean and SEMs for five replicates are indicated by symbols and error bars.

of A549 lung cancer cells (41). Troglitazone has also been shown to have antimigratory properties in glioma cells, associated with transcriptional repression of TGF $\beta(1-3)$ and their receptors I and II and with reduced TGF $\beta$ release (42) and to inhibit the TGF $\beta$-induced epithelial-mesenchymal transition of primary alveolar epithelial cells (43). On the basis of similarity in downregulated genes (Fig. 2), in particular the strong inhibition of the fibronectin 1 gene and integrin- $\beta 5$, our expectation is that our other candidate drugs would also exert their antimigratory effect through inhibition of TGF $\beta$ signaling. To demonstrate the effects of the three compounds on the TGF $\beta$ signaling pathway, we utilized a cell line stably expressing a reporter of TGF $\beta$ signaling (see Materials and Methods). The three compounds demonstrated significant inhibition of TGF $\beta$ signaling and are likely to inhibit cell migration and invasion with the same mode of action as resveratrol (Fig. 3), thus strengthening our predictions based on a global computational screen.

Experimental validation of citalopram, troglitazone, and enilconazole against metastatic colorectal cancer

We verified the predictions by in vitro tests showing that the three compounds troglitazone, citalopram, and enilconazole significantly inhibited cell migration and clonogenic survival of HCT 116 and HT-29 human colorectal cancer cells, both important hallmarks of metastasis (Fig. 4). Although we were specifically searching for antimetastatic compounds, we additionally sought to avoid growth stimulatory effects on primary tumors. For this purpose, the three compounds were tested for effects on proliferation. They did in fact inhibit this process albeit to a different extent (Fig. 4).

On the basis of the promising results of the in vitro assays, next we tested the three compounds in a subcutaneous tumor model in mice (Fig. 5A; ref. 44). We anticipated inhibition of tumor growth as all three compounds had demonstrated antiproliferative effects. Immunodeficient mice (NOD scid- $\gamma$ ) were subcutaneously implanted with tumor fragments and monitored for tumor growth. All three compounds significantly inhibited the growth of subcutaneous tumors, but enilconazole and citalopram showed the most profound effects (Fig. 5A). Of these two, citalopram was tolerated best by the mice and was investigated further. PI/Annexin-based FACS

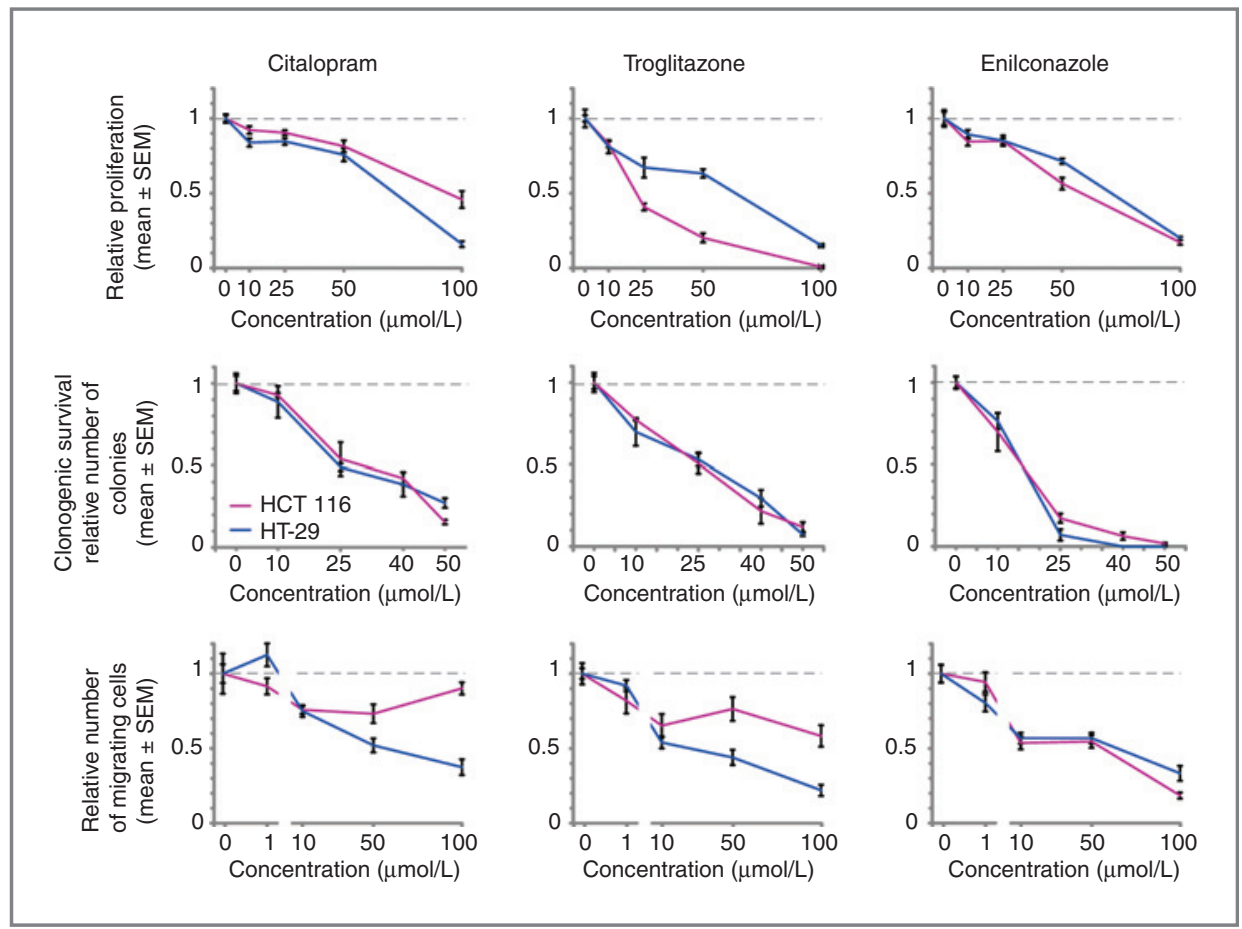

Figure 4. Inhibition of proliferation clonogenic survival, and migration of Human HCT 116 and HT-29 colorectal cancer cell lines. Graphs represent the mean and SEMs of relative proliferation, clonogenic survival rates, and relative number of migrating cells at different concentrations of citalopram, troglitazone, and enilconazole (see Materials and Methods). Proliferation assay, mean and SEMs for quadruplicate wells in increasing cell numbers per well $(250,500,1,000,2,000$ cells per well) are indicated by symbols and error bars. Migration and clonogenic survival assays, mean and SEMs for triplicate wells are indicated by symbols and error bars. 


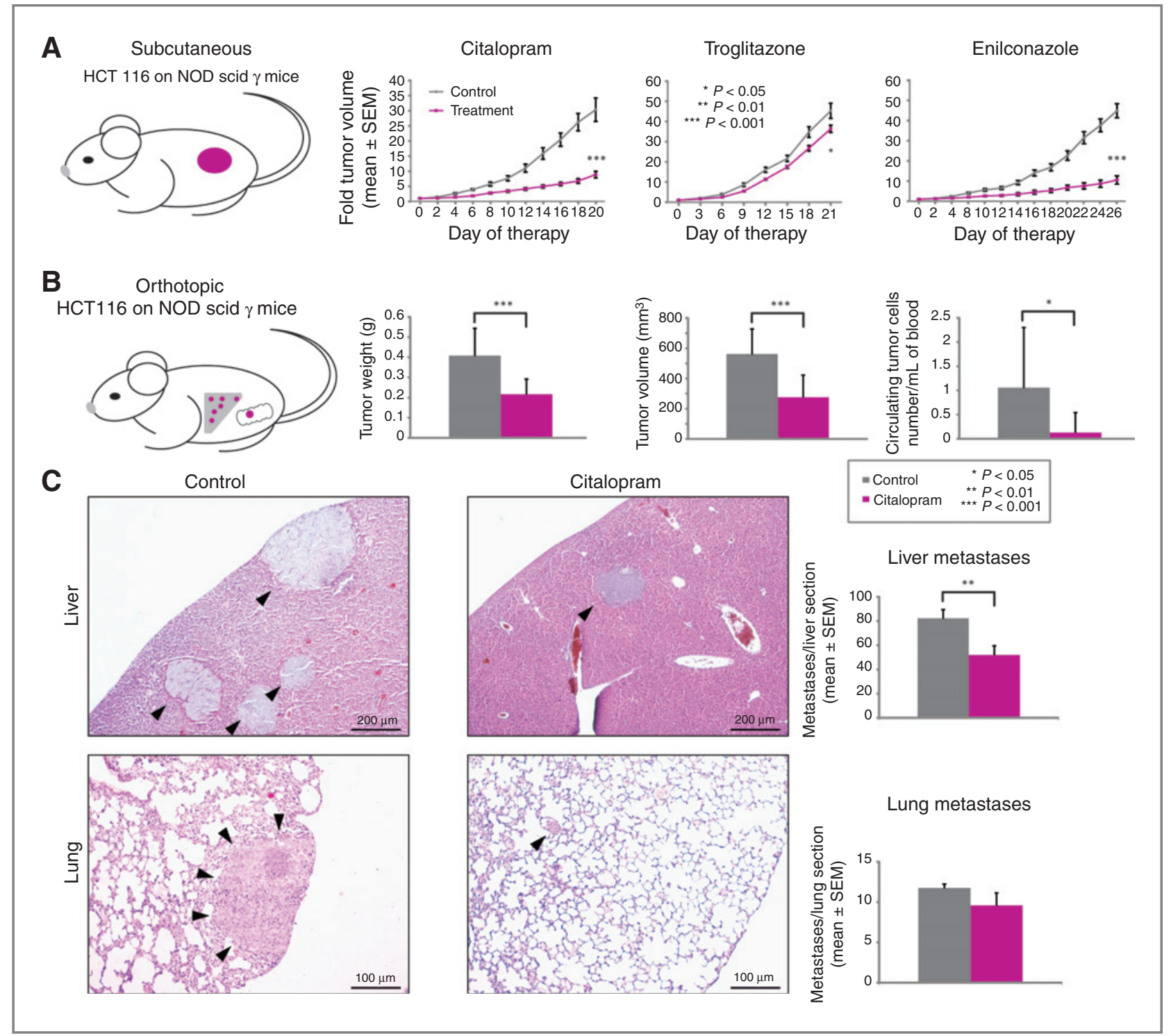

Figure 5. Inhibition of in vivo colorectal cancer tumor growth and colorectal cancer metastasis. A, immunodeficient (NOD scid gamma) mice were injected subcutaneously with HCT 116 colorectal cancer and treated with citalopram, troglitazone, or enilconazole or their respective vehicles (see Materials and Methods). Tumor volumes were measured in both treatments and control mice as indicated. B, NOD scid $\gamma$ mice were injected with HCT 116 colorectal cancer cells in the cecal wall and treated with either citalopram or vehicle ( $\mathrm{NaCl} 0.9 \%)$. After euthanasia, the total tumor size and volumes were measured. Blood was taken from control and treated mice and circulating tumor cells counted. C, histology of livers and lungs of

orthotopic colorectal cancer mice as in B. Exemplary pictures of hematoxylin and eosin stainings of livers and lungs of control animals (left) and citalopram-treated animals (middle). Histograms (right) show mean numbers of liver and lung metastases in control animals and citalopram-treated animals. In s.c. experiments, the symbols and error bars represent mean and SEM of 12 to 15 animals/group. In orthotopic experiments, data (mean \pm SEM) of 11 animals per group are depicted.

assays evaluating the fraction of apoptotic cells confirmed that the compounds were indeed inhibiting metastasis and not generally cytotoxic on tumors (Supplementary Fig. S3).

As subcutaneous tumor models primarily simulate growth of the primary tumor and can reproduce the metastatic cascade only to a limited extent, we next investigated citalopram in an orthotopic mouse model of colorectal cancer, which is able to reproduce the metastatic cascade and mimic the clinical course of colorectal cancer (44). We established an orthotopic model of colorectal cancer by surgical implantation of a tumor in the cecal wall (see Materials and Methods). Mice were treated with citalopram or vehicle. After 35 days, all mice in the control group were moribund due to extensive tumor burden, whereas the mice in the treatment group were in significantly better condition. The mice were sacrificed and tumor volume and tumor weight were measured, which were both significantly reduced in the citalopram group as compared with the controls (Fig. 5B). As a direct correlate of the metastatic activity of a tumor, we also quantified the CTC in the blood of the animals (Supplementary Fig. S4). CTC 
numbers were significantly reduced in the citalopram-treated mice, indicating a reduced metastatic activity of the tumors after citalopram treatment. Finally, the number of metastases in the livers and lungs were quantified and revealed a significantly reduced number of metastatic deposits in the livers of the animals (Fig. 5C; $P<0.01$ ). The number of metastatic nodules in the lungs was also reduced by citalopram treatment, however, failed to reach statistical significance $(P=0.27)$. These results show that citalopram can inhibit both tumor growth as well as metastasis of colorectal cancer.

\section{Discussion}

Using a system pharmacology approach based on the integration of publicly available drug-induced gene-expression data, we inferred that the antidiabetic drug troglitazone, the antifungal drug enilconazole, and the antidepressant citalopram could potentially lead to novel therapeutics for the prevention of metastasis in colorectal cancer, thereby reducing the leading cause of death in patients with colorectal cancer. On the basis of the known mode of action of the top correlated drugs and functional analysis of inhibited genes, we propose that the main mode of action of these compounds is via the inhibition of TGF $\beta$ signaling. We performed experimental validations for the anticancer activities of all three compounds using in vitro and subcutaneous tumor models in mice and we showed the efficacy of citalopram in an orthotopic mouse model of colorectal cancer, which is the clinically most relevant model to examine the effects of a compound on the process of metastasis.

In an orthotopic model, we demonstrated that citalopram had effects on both CTC numbers, the gold-standard surrogate marker for metastatic activity (45) as well as on the number of hepatic metastases, which is the clinically apparent outcome of a tumor's metastatic activity.

Because of low tolerability in the subcutaneous model, we could not test enilconazole and troglitazone in the orthotopic colorectal cancer model. Still, the in vitro experiments show the desired effect so that they represent promising leads to arrive at similar compounds, which are tolerated better and can be tested against metastatic colorectal cancer in the future.

Although the number of hepatic metastases was significantly reduced in the citalopram-treated orthotopic colorectal cancer mice, the number of pulmonary foci was reduced as well, although this effect failed to reach statistical significance. This can most likely be explained by the filter effect of the hepatic capillary bed. As previously shown, the number of circulating tumor cells is significantly reduced in the blood after having passed the liver (i.e., the hepatic veins) as compared with before passing the liver (i.e., in the portal vein;

\section{References}

1. Lamb J, Crawford ED, Peck D, Modell JW, Blat IC, Wrobel MJ, et al. The Connectivity Map: using gene-expression signatures to connect small molecules, genes, and disease. Science 2006;313:1929-35.

2. Iorio $F$, Tagliaferri $R$, di Bernardo $D$. Identifying network of drug mode of action by gene expression profiling. J Comput Biol 2009;16:241-51. ref. 46). This filter effect may have weakened the impact of the treatment on pulmonary metastasis.

The heterogeneity of cancers and especially colorectal cancer makes us hypothesize that even better novel therapies could be developed through systems pharmacology. There are many different subtypes, both genetically, such as microsatellite-stable versus unstable or BRAF-driven versus KRASdriven and histologically, such as serrated versus classical colorectal cancer (47). In the future, the subclassification and molecular characterization of these colorectal cancer subtypes into more specific diseases as well as the development of corresponding murine disease models that mimic the specific disease subtypes will allow for the discovery of better targeted, stratified therapies.

We conclude that our implementation of the reverse geneexpression profiling concept shows very promising results for the retrieval of novel candidate drugs; in particular, for the treatment of cancer-like disease as we have shown in our general analysis. Indeed, CMap employs cancer cell lines to establish drug-induced gene-expression profiles where many genes involved in cancer-relevant pathways are expressed. In the LINCS consortium, more data are being generated for various types of human primary cells and cell lines that will even improve the power of this approach (http://www. lincsproject.org). More relevant cell line or tissue models will make this approach more broadly applicable to other types of diseases in addition to cancer.

\section{Disclosure of Potential Conflicts of Interest \\ No potential conflicts of interest were disclosed.}

\section{Authors' Contributions}

Conception and design: V. van Noort, S. Schölch, M. Iskar, M. Koch, P. Bork Development of methodology: V. van Noort, S. Schölch, M. Iskar, G. Zeller, C. Schweitzer, K. Werner

Acquisition of data (provided animals, acquired and managed patients, provided facilities, etc.): S. Schölch, K. Ostertag, C. Schweitzer, J. Weitz Analysis and interpretation of data (e.g., statistical analysis, biostatistics, computational analysis): V. van Noort, S. Schölch, M. Iskar G. Zeller, K. Ostertag, C. Schweitzer, K. Werner

Writing, review, and/or revision of the manuscript: V. van Noort, S. Schölch, M. Iskar, G. Zeller, K. Ostertag, J. Weitz, M. Koch, P. Bork

Administrative, technical, or material support (i.e., reporting or organizing data, constructing databases): V. van Noort, S. Schölch, C. Schweitzer K. Werner

Study supervision: V. van Noort, S. Schölch, M. Koch, P. Bork

\section{Grant Support}

This work received funding through the CancerBiome project (European Research Council project reference 268985).

The costs of publication of this article were defrayed in part by the payment of page charges. This article must therefore be hereby marked advertisement in accordance with 18 U.S.C. Section 1734 solely to indicate this fact.

Received December 13, 2013; revised June 25, 2014; accepted July 3, 2014; published OnlineFirst July 18, 2014.
3. Dudley JT, Sirota M, Shenoy M, Pai RK, Roedder S, Chiang AP, et al. Computational repositioning of the anticonvulsant topiramate for inflammatory bowel disease. Sci Transl Med 2011;3:96ra76.

4. Sirota M, Dudley JT, Kim J, Chiang AP, Morgan AA, Sweet-Cordero A, et al. Discovery and preclinical validation of drug indications using 
compendia of public gene expression data. Sci Transl Med 2011; 3:96ra77.

5. Kunkel SD, Suneja M, Ebert SM, Bongers KS, Fox DK, Malmberg SE, et al. mRNA expression signatures of human skeletal muscle atrophy identify a natural compound that increases muscle mass. Cell Metab 2011;13:627-38.

6. Jahchan NS, Dudley JT, Mazur PK, Flores N, Yang D, Palmerton A, et al. A drug repositioning approach identifies tricyclic antidepressants as inhibitors of small cell lung cancer and other neuroendocrine tumors. Cancer Discov 2013;3:1364-77.

7. Iskar M, Campillos M, Kuhn M, Jensen LJ, van Noort V, Bork P. Drug-induced regulation of target expression. PLoS Comput Biol 2010;6.

8. Siegel R, Naishadham D, Jemal A. Cancer statistics, 2013. CA Cancer J Clin 2013;63:11-30.

9. Weitz J, Koch M, Debus J, Hohler T, Galle PR, Buchler MW. Colorectal cancer. Lancet 2005;365:153-65.

10. Cunningham D, Atkin W, Lenz HJ, Lynch HT, Minsky B, Nordlinger B, et al. Colorectal cancer. Lancet 2010;375:1030-47.

11. Vogelstein B, Papadopoulos N, Velculescu VE, Zhou S, Diaz LA Jr, Kinzler KW. Cancer genome landscapes. Science 2013;339:1546-58.

12. Rahbari NN, Aigner M, Thorlund K, Mollberg N, Motschall E, Jensen K, et al. Meta-analysis shows that detection of circulating tumor cells indicates poor prognosis in patients with colorectal cancer. Gastroenterology 2010;138:1714-26.

13. Pantel K, Riethdorf $S$. Pathology: are circulating tumor cells predictive of overall survival? Nat Rev Clin Oncol 2009;6:190-1.

14. Steinert G, Scholch S, Koch M, Weitz J. Biology and significance of circulating and disseminated tumour cells in colorectal cancer. Langenbecks Arch Surg 2012;397:535-42.

15. Cohen SJ, Punt CJ, lannotti N, Saidman BH, Sabbath KD, Gabrail NY, et al. Relationship of circulating tumor cells to tumor response, progression-free survival, and overall survival in patients with metastatic colorectal cancer. J Clin Oncol 2008;26:3213-21.

16. Steinert G, Scholch S, Niemietz T, Iwata N, Garcia SA, Behrens B, et al. Immune escape and survival mechanisms in circulating tumor cells of colorectal cancer. Cancer Res 2014;74:1694-704.

17. van der Bij GJ, Oosterling SJ, Beelen RH, Meijer S, Coffey JC, van Egmond $\mathrm{M}$. The perioperative period is an underutilized window of therapeutic opportunity in patients with colorectal cancer. Ann Surg 2009;249:727-34.

18. Lamb J. The Connectivity Map: a new tool for biomedical research. Nat Rev Cancer 2007;7:54-60.

19. Barrett T, Troup DB, Wilhite SE, Ledoux P, Evangelista C, Kim IF, et al. NCBI GEO: archive for functional genomics data sets-10 years on. Nucleic Acids Res 2011;39:D1005-10.

20. Butte AJ, Chen R. Finding disease-related genomic experiments within an international repository: first steps in translational bioinformatics. AMIA Annu Symp Proc 2006:106-10.

21. Dudley JT, Butte AJ. Identification of discriminating biomarkers for human disease using integrative network biology. Pac Symp Biocomput 2009:27-38.

22. Pacini C, lorio F, Goncalves E, Iskar M, Klabunde T, Bork P, et al. DvD: an $\mathrm{R} /$ Cytoscape pipeline for drug repurposing using public repositories of gene expression data. Bioinformatics 2013;29:132-4.

23. Irizarry RA, Bolstad BM, Collin F, Cope LM, Hobbs B, Speed TP. Summaries of Affymetrix GeneChip probe level data. Nucleic Acids Res 2003;31:e15.

24. Subramanian A, Tamayo $P$, Mootha VK, Mukherjee S, Ebert BL, Gillette MA, et al. Gene set enrichment analysis: a knowledge-based approach for interpreting genome-wide expression profiles. Proc Natl Acad Sci U S A 2005;102:15545-50.

25. Jorissen RN, Gibbs P, Christie M, Prakash S, Lipton L, Desai J, et al. Metastasis-associated gene expression changes predict poor outcomes in patients with dukes stage B and C colorectal cancer. Clin Cancer Res 2009;15:7642-51.

26. Abdollahi A, Lipson KE, Han X, Krempien R, Trinh T, Weber KJ, et al. SU5416 and SU6668 attenuate the angiogenic effects of radiationinduced tumor cell growth factor production and amplify the direct anti-endothelial action of radiation in vitro. Cancer Res 2003;63: 3755-63.

27. Huber PE, Bischof M, Jenne J, Heiland S, Peschke P, Saffrich R, et al. Trimodal cancer treatment: beneficial effects of combined antiangiogenesis, radiation, and chemotherapy. Cancer Res 2005;65:3643-55

28. Motulsky $\mathrm{HJ}$, Brown RE. Detecting outliers when fitting data with nonlinear regression - a new method based on robust nonlinear regression and the false discovery rate. BMC Bioinformatics 2006;7:123.

29. Qu XA, Rajpal DK. Applications of Connectivity Map in drug discovery and development. Drug Discov Today 2012;17:1289-98.

30. Ki DH, Jeung HC, Park CH, Kang SH, Lee GY, Lee WS, et al. Whole genome analysis for liver metastasis gene signatures in colorectal cancer. Int J Cancer 2007;121:2005-12.

31. Pantaleo MA, Astolfi A, Nannini M, Paterini P, Piazzi G, Ercolani G, et al. Gene expression profiling of liver metastases from colorectal cancer as potential basis for treatment choice. Br J Cancer 2008;99:1729-34.

32. Ganepola GA, Mazziotta RM, Weeresinghe D, Corner GA, Parish CJ, Chang $\mathrm{DH}$, et al. Gene expression profiling of primary and metastatic colon cancers identifies a reduced proliferative rate in metastatic tumors. Clin Exp Metastasis 2010;27:1-9.

33. Rousset N, Vonarx V, Eleouet S, Carre J, Kerninon E, Lajat Y, et al. Effects of photodynamic therapy on adhesion molecules and metastasis. J Photochem Photobiol B 1999;52:65-73.

34. Wu H, Liang X, Fang Y, Qin X, Zhang Y, Liu J. Resveratrol inhibits hypoxia-induced metastasis potential enhancement by restricting hypoxia-induced factor-1 alpha expression in colon carcinoma cells. Biomed Pharmacother 2008;62:613-21.

35. Luo XG, Zou JN, Wang SZ, Zhang TC, Xi T. Novobiocin decreases SMYD3 expression and inhibits the migration of MDA-MB-231 human breast cancer cells. IUBMB Life 2010;62:194-9.

36. Pankov R, Yamada KM. Fibronectin at a glance. J Cell Sci 2002; 115:3861-3.

37. Wang PS, Chou FS, Porchia L, Saji M, Pinzone JJ. Troglitazone inhibits cell migration, adhesion, and spreading by modulating cytoskeletal rearrangement in human breast cancer cells. Mol Carcinog 2008; 47:905-15

38. Aizawa J, Sakayama K, Kamei S, Kidani T, Yamamoto H, Norimatsu Y, et al. Effect of troglitazone on tumor growth and pulmonary metastasis development of the mouse osteosarcoma cell line LM8. BMC Cancer 2010;10:51.

39. Steffan JJ, Cardelli JA. Thiazolidinediones induce Rab7-RILP-MAPKdependent juxtanuclear lysosome aggregation and reduce tumor cell invasion. Traffic 2010;11:274-86.

40. Hocevar BA, Brown TL, Howe PH. TGF-beta induces fibronectin synthesis through a c-Jun N-terminal kinase-dependent, Smad4independent pathway. EMBO J 1999;18:1345-56.

41. Wang $H$, Zhang $H$, Tang $L$, Chen $H$, Wu C, Zhao M, et al. Resveratrol inhibits TGF-beta1-induced epithelial-to-mesenchymal transition and suppresses lung cancer invasion and metastasis. Toxicology 2013 303:139-46.

42. Coras R, Holsken A, Seufert S, Hauke J, Eyupoglu IY, Reichel M, et al. The peroxisome proliferator-activated receptor-gamma agonist troglitazone inhibits transforming growth factor-beta-mediated glioma cell migration and brain invasion. Mol Cancer Ther 2007;6:1745-54.

43. Zhou B, Buckley ST, Patel V, Liu Y, Luo J, Krishnaveni MS, et al. Troglitazone attenuates TGF-beta1-induced EMT in alveolar epithelial cells via a PPARgamma-independent mechanism. PLoS ONE 2012;7: e38827.

44. Guilbaud N, Kraus-Berthier L, Meyer-Losic F, Malivet V, Chacun C, Jan $\mathrm{M}$, et al. Marked antitumor activity of a new potent acronycine derivative in orthotopic models of human solid tumors. Clin Cancer Res 2001; 7:2573-80.

45. Pantel K, Alix-Panabieres $C$, Riethdorf S. Cancer micrometastases. Nat Rev Clin Oncol 2009;6:339-51.

46. Rahbari NN, Bork U, Kircher A, Nimitz T, Scholch S, Kahlert C, et al Compartmental differences of circulating tumor cells in colorectal cancer. Ann Surg Oncol 2012;19:2195-202.

47. Comprehensive molecular characterization of human colon and rectal cancer. Nature 2012;487:330-7. 


\section{Cancer Research \\ ACR \\ Novel Drug Candidates for the Treatment of Metastatic Colorectal Cancer through Global Inverse Gene-Expression Profiling}

Vera van Noort, Sebastian Schölch, Murat Iskar, et al.

Cancer Res 2014;74:5690-5699. Published OnlineFirst July 18, 2014.

Updated version Access the most recent version of this article at: doi:10.1158/0008-5472.CAN-13-3540

Supplementary Access the most recent supplemental material at:

Material http://cancerres.aacrjournals.org/content/suppl/2014/07/21/0008-5472.CAN-13-3540.DC1.html

Cited Articles This article cites by 44 articles, 18 of which you can access for free at:

http://cancerres.aacrjournals.org/content/74/20/5690.full.html\#ref-list-1

E-mail alerts Sign up to receive free email-alerts related to this article or journal.

Reprints and To order reprints of this article or to subscribe to the journal, contact the AACR Publications Department at Subscriptions pubs@aacr.org.

Permissions To request permission to re-use all or part of this article, contact the AACR Publications Department at permissions@aacr.org. 